\title{
SOMACLONAL VARIATION IN RESISTANCE OF PERENNIAL RYEGRASS TO CROWN RUST
}

\author{
R. A. Skipp and D. W. R. White
}

Plant Diseases Division and Grasslands Division, DSIR, Palmersion North

\begin{abstract}
Resistance of perennial ryegrass plants regenerated from tissue culture (somaclones) to crown rust (Puccinia coronata) was assessed in growth cabinet/glasshouse experiments. Somaclones regenerated from callus cultures initiated from the vegetative tissue of three different somaclonal parent plants were tested for resistance to crown rust collected from Palmerston North and Lincoln. Most plants tested were highly resistant and their leaves developed small pale yellow flecks but showed little or no sporulation. However, a few large pustules did develop on leaves of some of the somaclones. Screening experiments with rust cultures initiated from each of three single pustules showed that somaclones varied in their reaction to crown rust. Wiiin each group of somaclones derived from a particular parent some were more susceptible. and some more resistant, than the parental genotype.

Two of the somaclones obtained from one of the parent plants had very fine leaves similar to those of turf perennial ryegrass plants. Somacloning may provide an efficient technique for plant breeders to improve the crown rust resistance of both pasture and turf perennial ryegrasses.
\end{abstract}

Keywords: Puccinia coronata, Lolium perenne, somaclones. tissue culture, plant breeding, plant diseases.

\section{INTRODUCTION}

Perennial ryegrass in pasture often becomes heavily infected with crown rust (caused by Puccinia coronata) in late summer and autumn. Rust infection reduces ryegass growth which in turn encourages clover dominance and the ingress of weeds (Lancashire \& Latch 1966, 1970). Rust infected leaves rapidly become senescent and die. This reduces the palatability and nutritional value of the herbage and increases the danger of facial eczema in grazing sheep (McKenzie 1971).

There is some natural resistance to crown rust within perennial ryegrasses (Wilkins 1975; Kopec et al. 1983). However, although there has been some selection for resistance in breeding programmes in New Zealand (Lancashire \& Latch 1966) and elsewhere (Braverman 1986), perennial ryegrass cultivars grown in New Zealand have remained highly susceptible to rust.

White (this proceedings) found that plants regenerated from tissue cultures (somaclones) from a single immature embryo of perennial ryegrass showed considerable morphological variation and were highly resistant to crown rust. This resistance was heritable. Plant material grown in tissue culture can undergo extensive genetic alteration in various heritable characteristics, including disease resistance (Daub 1986); however, for reasons discussed elsewhere (White this proceedings) is was not clear whether the rust resistance of the perennial ryegrass somaclones: (1) had increased or decreased compared with that of the parent plant, or (2) was race specific or race non-speoific. Such information would be valuable if this resistance were to be exploited in a breeding programme.

Three of the somaclones were subjected to a further cycle of tissue culture and plant regeneration (White this proceedings). The three somaclonal lines thus produced allowed comparison of the newly regenerated somaclones with their corresponding somaclonal parents. This paper reports on the resistance of these somaclonal lines to infection by $\boldsymbol{P}$. coronata.

\section{MATERIALS ANO METHODS}

The perennial ryegrass plants used for these experiments comprised three somaclonal parent plants (designated 96PR, 108PR, and 126PR) which had been regenerated from 
tissue culture of a single callus line (SR 1; White 1987), and 52 somaclones. The somaclones (29, 11, and 12 plants respectively from the parents 96PR, 108PR, and 126PR) had been regenerated from callus cultures of immature inflorescences of the somaclonal parents (White this proceedings).

Five vegetative clones were propagated from each plant and grown in a peat/sand compost in $10-\mathrm{cm}$ pots in the glasshouse $\left(14-16^{\circ} \mathrm{C}\right)$. A polyethylene sleeve $(35 \mathrm{~cm}$ long) was fitted to the top of each pot after inoculation to provide high humidity for infection and to restrict cross contamination of rust cultures.

Crown rust was collected from perennial ryegrass plants in field plots at DSIR Grasslands Division (two collections from Palmerston North and one from Lincoln). The three mass collections were cultured separately on Grasslands Ruanui perennial ryegrass plants in growth cabinets $\left(20^{\circ} \mathrm{C} ; 16 \mathrm{~h}\right.$ day). Cultures of single-pustule isolates were initiated by inoculating Ruanui plants with urediospores from individual pustules on somaclones infected with crown rust collected in Palmerston North. Urediospores produced by the rust cultures were collected on aluminium foil placed around the base of plants, and stored in petri dishes at $4^{\circ} \mathrm{C}$.

In each screening test, urediospores from a single rust culture were used to inoculate one plant of each of the somaclonal parents and of their respective somaclones. A Ruanui plant served as a susceptible control. Urediospore inoculum was diluted 1:30 (w/w) with talc and brushed on to the adaxial surfaces of leaves. Water was atomised on to plants and the polyethylene sleeves closed for $24 \mathrm{~h}$. Plants were kept in the growth cabinets for 7 days after inoculation then transferred to the glasshouse for a further 7 days. Infection type was then scored according to the grading system: 0 , no pustules or other symptoms; 1 , no pustules chlorotic flecks present; 2 , minute pustules - chlorotic flecks present; 3 , small pustules surrounded by a band of chlorotic tissue; 4 , medium-sized pustules - some chlorosis; 5 , large pustules - little chlorosis. The highest infection type on each plant was recorded.

\section{RESULTS AND DISCUSSION}

\section{Screening experiments with mass collections of crown rust}

The somaclonal parents and most somaclones appeared highly resistant to the three mass collections of crown rust from Palmerston North and Lincoln (Fig. 1). Generally there was little sporulation on these plants (infection type $<3$ ). Infection type was lower on plants of the somaclonal lines than on those of the susceptible cultivar Ruanui. There was, however, considerable variation in infection type among somaclones, which suggested that individual somaclones differed in their susceptibility to crown rust. For instance, the mean infection type on 10 of the somaclones was 1 or less, indicating that they had a high level of resistance to all three cultures, while two plants of line 108 were moderately susceptible to the two Palmerston North cultures which resulted in higher mean infection type scores $(<2.5)$. Some somaclones exhibited markedly lower or higher infection types than their corresponding somaclonal parents.

Symptoms that developed on leaves of somaclones at the sites of individual infections also varied; infection type sometimes ranged from 1 to 4 (i.e., from no sporulation to medium-sized pustules) on a single plant. This suggested that the cultures of rust from the mass collections were genetically heterogeneous with respect to their virulence on the ryegrass somaclones, and probably consisted of a mixture of physiological races (Wilkins $1975,1978)$. Detailed studies on the nature of somaclonal variation in resistance to crown rust would require the use of genetically homogeneous rust cultures such as those derived from single pustules.

\section{Screening experiments with single pustule isolates of crown rust}

Single-pustule isolates of crown rust from susceptible Ruanui plants which had been inoculated with a mass collection of rust from Palmerston North gave a uniform infection type 


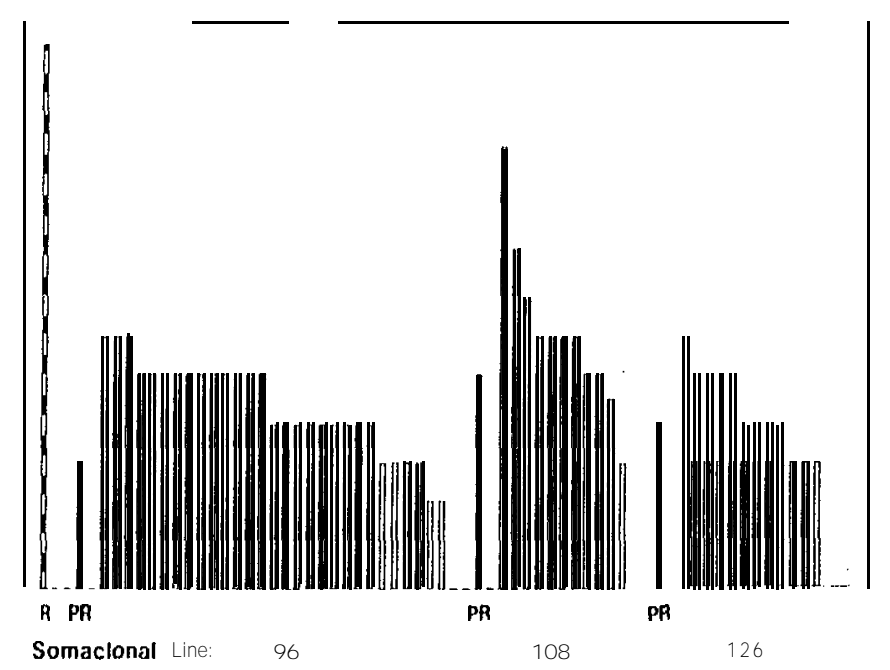

Figure 1: Mean infection type of three mass collections of Puccinia coronata from Palmerston-North and Lincoln on a susceptible cultivar, Grasslands Ruanui (R), and three somaclonal lines (96. 108 and 126) each dewed from a separate somaclonal parent (PR).

of 1 on all somaclones and their parents; thus they failed to distinguish any inherent differences among somaclones in their resistance to crown rust. However, cultures initiated from some large pustules which appeared on several somaclones of lines 96, 108, and 126 in the mass collection screening experiments described above proved more promising. Some of these cultures gave a uniformly high infection type when reinoculated on to the somaclone from which they had been obtained and a uniformly low infection type on some other somaclones. Three of such cultures were used for further testing.

As found in experiments with mass collections of crown rust, most plants of the somaclonal lines were resistant to the three single-pustule isolates (Fig. 2). These isolates gave low but variable infection type scores on the somaclonal parents and somaclones. Somaclones of line 96 gave predominantly lower infection types than their somaclonal parent, whereas somaclones of lines 108 and 126 gave predominantly higher infection types.

Infection type on most individual somaclones varied by no more than 1 in successive tests with the three different single-pustule isolates. This was similar to the range of infection type exhibited by the different vegetative clones of individual somaclones (unpublished data). However, 11 somaclones exhibited a range in infection type of 2 or more (e.g. from type 1 or 2 to 4), thus appearing resistant to one or more isolate but susceptible to the others. These results suggested that the single pustule isolates used represented different physiological races of $P$. coronata, and that the somaclones giving a variable response to different isolates could possess a major gene for resistance to one of the races.

\section{General Discussion}

Evidence that there are biotypes or physiological races of $P$. coronata which differ in their virulence on particular ryegrass genotypes has come from other work (Wilkins 1978). Different races appear to predominate in different countries as can be inferred from reports that Ruanui, which is very susceptible to crown rust in New Zealand, is among the most resistant of cultivars tested in the USA (Kopec et al. 1983). Genetic control of rust resistance in perennial ryegrass can be both monogenic or oligogenic (usually qualitative and race specific), and multigenic (usually quantitative and race non-specific) (Wilkins 1975, 1978; Hayward 1977). We have as yet little information about the genetic control of resistance in 


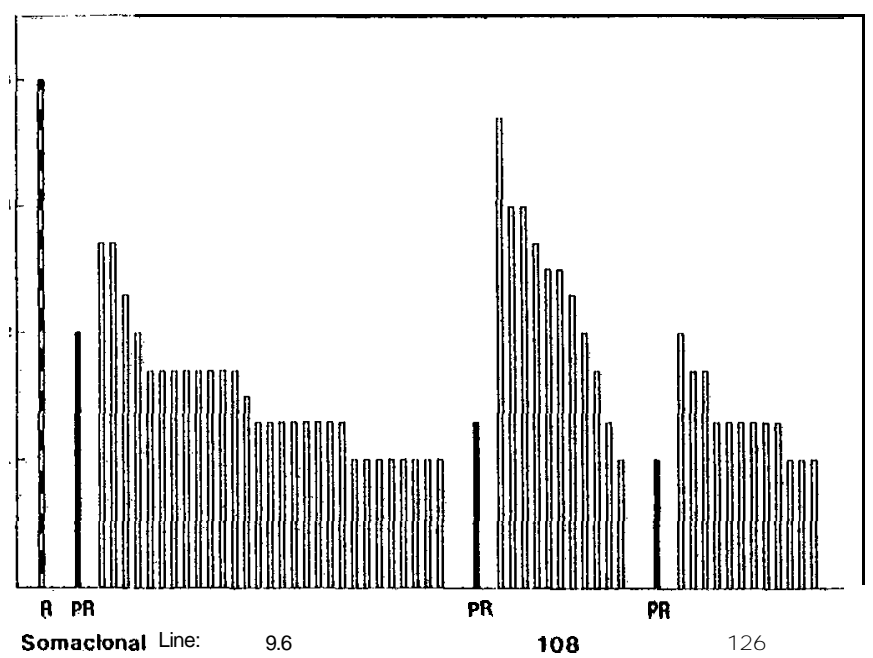

Figure 2: Mean infection type of three single pustule isolates of Puccinia coronata on a susceptibte cultivar, Grasslands Ruanui (R), and three somaclonal lines (96. 106 and 126) each derived from a separate somaclonal parent (PR).

the ryegrass somaclones, or even an indication whether resistance is genetically or physiologically similar to that found in non-somaclonal ryegrass. Further use of single pustule isolates of $P$. coronata in infection studies with selected somaclones could be informative in this respect, and could help elucidate the complexity of rust races in New Zealand.

Two of the somaclones derived from somaclonal parent 108 used in this study had short and fine leaves similar to those of turf perennial ryegrass plants. This finding emphasises that somacloning can generate variation in more than one character. Where combinations of several characters such as rust resistance and fine leaves are required, somacloning could increase. the efficbncy of a number of steps in the breeding process.

\section{References}

Braverman S.W. 1966. Disease resistance in cool season forage range and turt grasses. Botanical Review 52: 1-1 12. Daub M.E. 1966. Tissue culture and the selection of resistance to pathogens. Annual Review of Phytopathology 24: 159- 66.

Hayward M.D. 1977. Genetic control of resistance to crown rust (Puccinia coronata Corda) in ryegrass (Loliumperenne L.) and its, implications in breeding. Theoretical and Applied Genetics 51: 49-53.

Kopec D.M., Funk C.R., Haliski P.M. 1983. Sources and distribution of resistance to crown rust within perennial ryegrass. Plant Disease 67: 98-100.

Lancashire J.A., Latch G.C.M. 1966. Some effects of crown rust (Puccinia Coronata) on the growth of two ryegrass varieties in New Zealand. NZ Journat of Agricultural'Research 9: 628-640.

Lancashire J.A., Catch G.C.M. 1970. The effect of crown rust (Puccinia coronata Corda) on the yield and botanical composition of two ryegrass/white clover pastures. NZ Journal of Agricultural Research 13: 279-286.

McKenzie E.H.C. 1971. Seasonal changes in fungal spore numbers in ryegrass-white clover pasture, and effects of benomyl on pasture fungi, NZ Journal of Agricultural Research 14: 379-392.

Wilkins P.W. 1975. Implications of host-pathogen variation for resistance breeding in the grass crop. Annals of Applied Biology 81: 257-261.

Wilkins P.W: 1978: Specialisation of crown rust. on. highly and moderately resistant plants of perennial ryegrass. Annals of Applied:Biology 88: 179:184. 